\title{
Editorial: Quantitative Phase Imaging and Its Applications to Biophysics, Biology, and Medicine
}

\author{
YongKeun Park ${ }^{1,2 *}$, Gabriel Popescu ${ }^{3}$, Pietro Ferraro ${ }^{4}$ and Björn Kemper ${ }^{5}$ \\ ${ }^{1}$ Department of Physics, Korea Advanced Institute of Science \& Technology, Daejeon, South Korea, ${ }^{2}$ Tomocube Inc., \\ Daejeon, South Korea, ${ }^{3}$ Department of Electrical and Computer Engineering, University of Illinois at Urbana-Champaign, \\ Champaign, IL, United States, ${ }^{4}$ CNR - Institute of Applied Sciences \& Intelligent Systems Pozzuoli, Naples, Italy, ${ }^{5}$ Biomedical \\ Technology Center, University of Münster, Münster, Germany
}

Keywords: quantitative phase imaging, refractive index, label-free, microscopy, imaging

\section{Editorial on the Research Topic}

\section{Quantitative Phase Imaging and Its Applications to Biophysics, Biology, and Medicine}

Label-free imaging capability has played an important role throughout the history of microscopy, and it is also crucial for many research fields, including neuroscience and stem cell studies. From the invention of phase-contrast microscopy by Zernike [1], the refractive index has been exploited for label-free high-contrast imaging of unlabeled live biological cells and tissues. The development of a series of label-free imaging techniques, including differential interference microscopy [2] and reflection interference contrast microscopy [3], has dramatically expanded the applicability of microscopy for the precise investigation of the morphology of cells and subcellular organelles.

Recent developments in quantitative phase imaging (QPI) [4] have highly expanded the applicability of the refractive index as a reporter for advanced biological studies [5]. By directly and quantitatively measuring refractive index distributions or optical phase delay information, QPI provides various pieces of morphological and biophysical information about live cells and tissues, generating a series of new methods for cell biology [6, 7], biophysics [8], reproductive science [9-11], infectious diseases [12], hematology [10, 13], and neuroscience [14, 15].

This Special Research Topic includes a collection of research results that push the frontiers of QPI to new areas and applications. The articles collected in this Research Topic can be categorized into three classes. The first sub-topic introduces new optical developments in QPI (Linarès-Loyez et al.; Lu et al.). The second sub-topic presents novel experimental methodology exploiting refractive index information (Bélanger et al.; Cohoe et al.). The third sub-topic features applications in biology and medicine (Hu et al.; Murray et al., Memmolo et al.; Yaikova et al.).

The first sub-topic starts with a study that presents a method for live super-resolution imaging and single-particle tracking in 3D. Linarès-Loyez et al. present a method that utilizes quantitative intensity and phase imaging in the formation of fluorescent self-interference. Lu et al. demonstrate a simple but powerful QPI method using an optical diffuser. By exploiting optical memory effects, the quantitative phase information is retrieved from a measurement of speckle patterns.

The papers in the second sub-topic present new approaches that utilize QPI. Bélanger et al. report an experimental method for measuring cell volumes using QPI and a low-cost, open-source, and 3D-printed flow chamber. Cohoe et al. demonstrate a label-free imaging approach for the study of protozoa. The optical phase delay images were measured at multiple wavelengths, which were utilized for effectively addressing a phase unwrapping issue in QPI.

The third sub-topic features new research results in the study of biology and medicine. Memmolo et al. present biophysical studies of red blood cells (RBCs) using QPI and a microfluidic 
device. RBCs demonstrate remarkable deformability, which is crucial for them to pass through small capillaries in physiological conditions. The deformability of RBCs is strongly correlated with the pathophysiology of various diseases, but access to it requires complicated and low-throughput conventional methods. In this work, the shapes of RBCs and their responses to flow conditions were quantitatively measured and analyzed. Hu et al. report measurements of collagen fiber organization in tissue using spatial light interference microscopy. They compared the collagen fiber organization in tissue affected by pelvic organ prolapse to asymptomatic controls ( $\mathrm{Hu}$ et al.). Murray et al. address an important problem in cancer diagnosis and treatment using QPI. They present a QPI-based in vitro drug screening method for identifying evolving resistance at an early stage in order to help make better clinical decisions for cancer therapies. Yaikova et al. present an algorithm to analyze bone tissue images by automatically differentiating

\section{REFERENCES}

1. Zernike F. How I discovered phase contrast. Science. (1955) 121:345-9. doi: 10.1126/science.121.3141.345

2. Allen RD, David GB, Nomarski G. The Zeiss-Nomarski differential interference equipment for transmitted-light microscopy. Z Wiss Mikrosk. (1969) 69:193-221.

3. Gingell D, Todd I. Interference reflection microscopy. A quantitative theory for image interpretation and its application to cellsubstratum separation measurement. Biophys J. (1979) 26:507-26. doi: 10.1016/S0006-3495(79)85268-6

4. Popescu G. Quantitative Phase Imaging of Cells and Tissues. New York, NY: McGraw-Hill (2011).

5. Park Y, Depeursinge C, Popescu G. Quantitative phase imaging in biomedicine. Nat Photon. (2018) 12:578-89. doi: 10.1038/s41566-018-0253-x

6. Kemper B, Von Bally G. Digital holographic microscopy for live cell applications and technical inspection. Appl Opt. (2008) 47:A52-A61. doi: 10.1364/AO.47.000A52

7. Kim TK, Lee BW, Fujii F, Lee KH, Lee S, Park Y, et al. Mitotic chromosomes in live cells characterized using high-speed and label-free optical diffraction tomography. Cells. (2019) 8:1368. doi: 10.3390/cells8111368

8. Park Y, Best CA, Badizadegan K, Dasari RR, Feld MS, Kuriabova T, et al. Measurement of red blood cell mechanics during morphological changes. Proc Natl Acad Sci USA. (2010) 107:6731-6. doi: 10.1073/pnas.0909533107

9. Di Caprio G, Ferrara MA, Miccio L, Merola F, Memmolo P, Ferraro P, et al. Holographic imaging of unlabelled sperm cells for semen analysis: a review. J Biophotonics. (2015) 8:779-89. doi: 10.1002/jbio.201400093

10. Nguyen TH, Kandel ME, Rubessa M, Wheeler MB, Popescu G. Gradient light interference microscopy for 3D imaging of unlabeled specimens. Nat Commun. (2017) 8:210. doi: 10.1038/s41467-017-00190-7 objects based on their image signals and recovering their morphological topology.

In sum, this Research Topic features eight excellent research reports encompassing the methodology of QPI and its applications in biology and medicine. The editors appreciate the contributions of all of the authors. Every year, an increasing number of interesting research papers in QPI have been reported, ranging from new optical methodologies and biological applications to the potential for clinical diagnosis. It is our hope that this special topic will further accelerate the scientific advancements in QPI and its practical applications in biology and medicine.

\section{AUTHOR CONTRIBUTIONS}

All authors listed have made a substantial, direct and intellectual contribution to the work, and approved it for publication.

11. Jiang H, Kwon JW, Lee S, Jo YJ, Namgoong S, Yao XR, et al. Reconstruction of bovine spermatozoa substances distribution and morphological differences between Holstein and Korean native cattle using three-dimensional refractive index tomography. Sci Rep. (2019) 9:8774. doi: 10.1038/s41598-01945174-3

12. Park Y, Diez-Silva M, Popescu G, Lykotrafitis G, Choi W, Feld MS, et al. Refractive index maps and membrane dynamics of human red blood cells parasitized by Plasmodium falciparum. Proc Natl Acad Sci USA. (2008) 105:13730-5. doi: 10.1073/pnas.0806100105

13. Popescu G, Park Y, Choi W, Dasari RR, Feld MS, Badizadegan K. Imaging red blood cell dynamics by quantitative phase microscopy. Blood Cells Mol Dis. (2008) 41:10-6. doi: 10.1016/j.bcmd.2008.01.010

14. Marquet P, Depeursinge C, Magistretti PJ. Exploring neural cell dynamics with digital holographic microscopy. Annu Rev Biomed Eng. (2013) 15:407-31. doi: 10.1146/annurev-bioeng-071812-152356

15. Cintora P, Arikkath J, Kandel M, Popescu G, Best-Popescu C. Cell density modulates intracellular mass transport in neural networks. Cytometry A. (2017) 91:503-9. doi: 10.1002/cyto.a.23111

Conflict of Interest: The authors declare that the research was conducted in the absence of any commercial or financial relationships that could be construed as a potential conflict of interest.

Copyright (c) 2020 Park, Popescu, Ferraro and Kemper. This is an open-access article distributed under the terms of the Creative Commons Attribution License (CC BY). The use, distribution or reproduction in other forums is permitted, provided the original author(s) and the copyright owner(s) are credited and that the original publication in this journal is cited, in accordance with accepted academic practice. No use, distribution or reproduction is permitted which does not comply with these terms. 